\title{
Diversidade de morcegos (Chiroptera, Mammalia) em fragmentos florestais no estado do Paraná, Brasil
}

\author{
Nelio Roberto dos Reis ${ }^{1}$ \\ Adriano Lúcio Peracchi ${ }^{2}$ \\ Margareth Lumy Sekiama ${ }^{3}$ \\ Isaac Passos de Lima ${ }^{1}$
}

\begin{abstract}
Diversity of bats (Chiroptera, Mammalia) in forest fragments. The bay of the Tibagi river, a place which goes through quick environmental alterations, has three forest fragments: the "Floresta Nacional do Irati" (3.572 ha), the "Reserva Biológica da Klabin" (11.116 ha) and the "Parque Estadual Mata dos Godoy" (680 ha). Here, Myotis ruber (E. Geoffroy, 1806) and Chiroderma doriae Thomas, 1891 can be found, these are threatened bats, and three species, Pygoderma bilabiatum (Wagner, 1843), Myotis levis (I. Geoffroy, 1824) and Rogheessa tumida H. Allen, 1866 , which are presumably threatened. Using the same effort of capture in the three places, it was stated that the "Floresta Nacional do Irati" with low vegetation diversity, showed nine species of bats. The "Parque Estadual Mata dos Godoy", although it's five times smaller, fifteen species. We came to the conclusion that a big reserve, without good conditions would house a lower number of species. A small reserve with adequate resources and great vegetation diversity presents a higher number of niches, housing a higher number of species.

KEY WORDS. Chiroptera, bats, diversity, forest fragments
\end{abstract}

As unidades de conservação protegem valores ambientais representativos, por isso teriam que assegurar o bem estar das comunidades animais e vegetais, melhorando as condições do ambiente na região onde estão localizadas, sem inibir o progresso econômico das localidades que as abrigam. Deveriam também assegurar uma proteção legal, mantendo ao máximo os valores naturais que justifiquem sua preservação, e ainda, ser uma unidade biogeográfica representativa com "habitats" favoráveis que preservem a flora e a fauna local.

Poderiam atender, como centros de educação e pesquisa, visando otimizar a convivência animal - floresta, em situações que preservem os "habitats" e evitem o risco de extinguir espécies, conseqüentemente proteger os materiais genéticos.

Todavia, essas regiões estão sofrendo alterações ambientais rápidas e significativas, devido ao seu potencial agropecuário, demandando cada vez mais apro-

1) Departamento de Biologia Animal e Vegetal, Universidade Estadual de Londrina. 86051-990 Londrina, Paraná, Brasil.

2) Departamento de Zoologia, Instituto de Biologia, Universidade Federal Rural do Rio de Janeiro. 23851-970 Seropédica, Rio de Janeiro, Brasil.

3) Departamento de Zoologia, Universidade Federal do Paraná. Caixa Postal 19020, 81531-990 Curitiba, Paraná, Brasil. Bolsista CNPq. 
priações inadequadas dessas áreas e de seus recursos regionais. A bacia do Rio Tibagi, com aproximadamente $25.000 \mathrm{~km}$, apresenta três grandes unidades de conservação: uma, no Alto Tibagi (Floresta Nacional do Irati, com 3.572 ha), outra, no Médio Tibagi (Reserva Biológica da Klabin, com 11.116 ha) e por fim, no Baixo Tibagi (Parque Estadual Mata dos Godoy, com 680 ha).

Nessas localidades encontram-se endemismos, também espécies raras que aos poucos estão desaparecendo. Dentro deste contexto, consideram-se que duas espécies de morcegos, Myotis ruber (E. Geoffroy, 1806) e Chiroderma doriae Thomas, 1891 encontram-se ameaçadas de extinção, segundo portaria 062 de 17 de junho de 1997 do IBAMA, e três espécies, Pygoderma bilabiatum (Wagner, 1843), Myotis levis (I. Geoffroy, 1824) e Rhogessa tumida H. Allen, 1866, estão presumivelmente ameaçadas de extinção. Os morcegos representam quase $25 \%$ da população de mamíferos de qualquer região tropical (HANDLEY 1966; NowAK 1991), com importância na recuperação de ecossistemas florestais (HUBER 1910; VAN DER PIJl 1957; HUMPHREY \& BONACCORSO 1979), sendo ainda, formadores iniciais de novas florestas. Este estudo teve como objetivo o de conhecer as espécies de morcegos que ocorrem nas unidades de conservação da Bacia do Rio Tibagi na esperança de proteger estes fragmentos florestais, esperando mostrar a importância desses para a preservação adequada da fauna e da flora nativa da região. Assegurando assim a proteção dos quirópteros nos seus "habitats". Apesar das unidades apresentarem um número representativo de espécies, a maioria delas são sensíveis a flutuações ambientais.

\section{MATERIAL E MÉTODOS}

\section{Descrição das áreas de estudo}

As três áreas de estudo estão localizadas na Bacia do Rio Tibagi. (Fig. 1), a saber:

\section{Parque Estadual Mata dos Godoy (Baixo Tibagi)}

O Parque conta com uma área de 680 ha e sua coordenada geográfica é $23^{\circ} 27^{\prime} \mathrm{S}$ e $51^{\circ} 15^{\prime} \mathrm{W}$. Sua altitude média é de $700 \mathrm{~m}$. O clima segundo a classificação de Köppen (TREWARTHA \& HORN 1990) é subtropical úmido. A temperatura média do mês mais frio é de $17.6^{\circ} \mathrm{C}$ e a temperatura do mês mais quente é de $24.5^{\circ} \mathrm{C}$. A precipitação pluviométrica média anual é de $1250 \mathrm{~mm}$, cortado pelo Trópico de Capricórnio. Apresenta uma floresta densa, subtropical, relativamente alta, recobrindo quase toda extensão do Parque. A floresta é basicamente latifoliar, com $20 \%$ de espécies decíduas e a altura média é de $25 \mathrm{~m}$ com árvores emergentes de 35 a $40 \mathrm{~m}$, com grande quantidade de lianas. A espécie com maior número de indivíduos é Aspidosperma polineurom (peroba). As de maior biomassa: A. polineurom, Ficus insipida (figueira-branca) e Croton floribundus (capixingui).

\section{Parque Ecológico da Klabin - Fazenda Monte Alegre (Médio Tibagi)}

A Fazenda Monte Alegre conta com uma área de $126.373,10$ ha e a sua coordenada geográfica é $24^{\circ} 12^{\prime} 42^{\prime \prime} \mathrm{S}$ e $50^{\circ} 33^{\prime} 26^{\prime \prime} \mathrm{O}$. A altitude média é de $885 \mathrm{~m}$ e o clima, segundo a classificação de Köppen (TREWARTHA \& HORN 1990), enqua- 
dra-se como subtropical com a temperatura média do mês mais frio de $16,3^{\circ} \mathrm{C}$ e a temperatura média do mês mais quente $23,2^{\circ} \mathrm{C}$. A precipitação pluviométrica média anual é de $1.478 \mathrm{~mm}$.

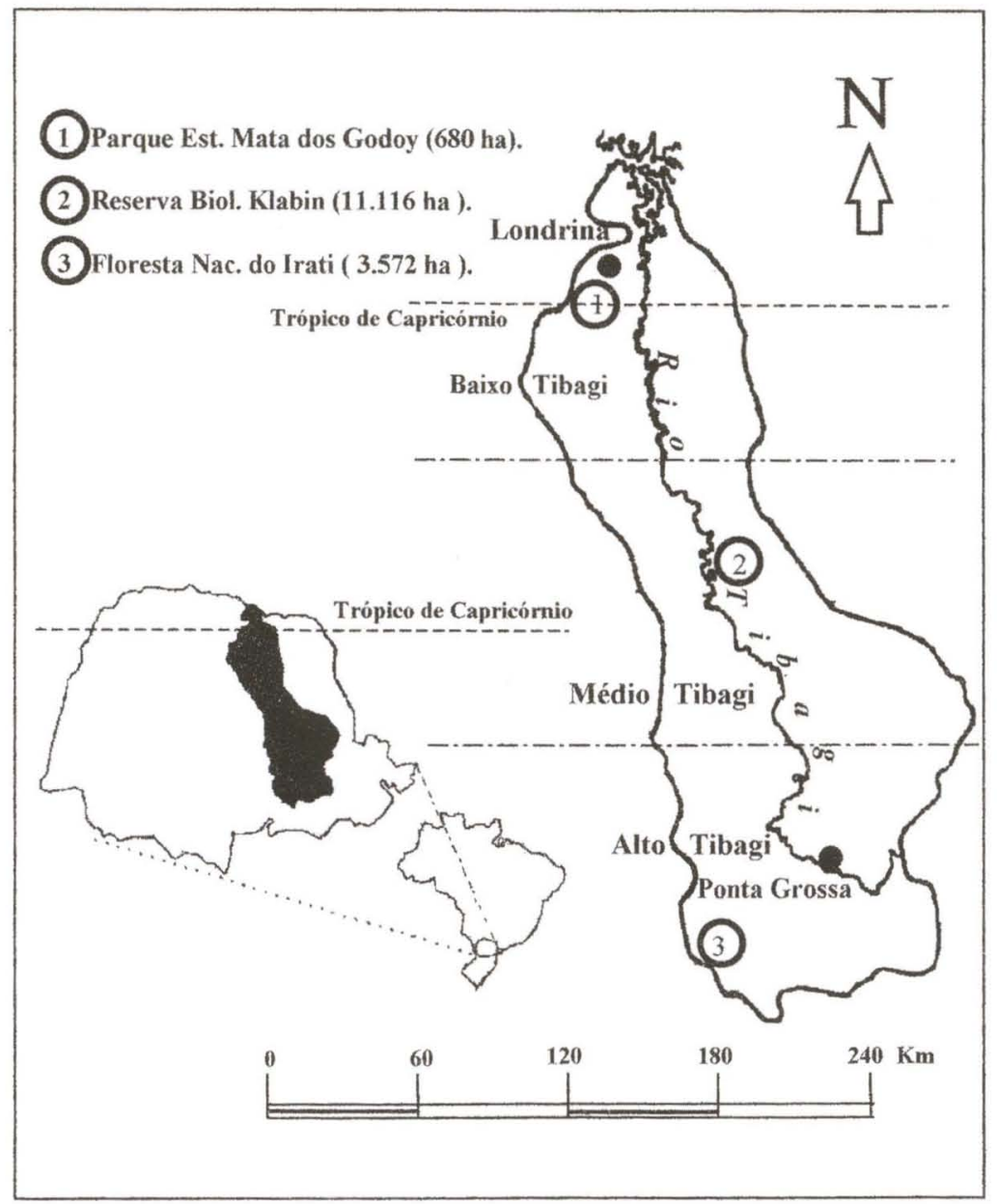

Fig. 1. Localização dos três fragmentos florestais estudados na Bacia do Rio Tibagi.

O Parque ocupa uma área de 11.116 ha, das quais 7.883 ha são representados por florestas naturais, algumas delas ainda em estado primitivo. A parte de floresta primária é constituída de árvores altas, destacando-se excepcionais exemplares de Araucaria angustifolia (pinheiro-do-Paraná), Patagonuta americana L. (guajavira) e Eugenia envolucrata DC. (cerejeira). O sub-bosque é dominado por samambaias, bromélias, cipós e avencas. 


\section{Floresta Nacional do Irati (Alto Tibagi)}

Ocupa uma área total de 3.571 ha, na coordenada geográfica $25^{\circ} 27^{\prime} \mathrm{S}$ e $50^{\circ} 35^{\prime} \mathrm{W}$. A altitude média é de $893 \mathrm{~m}$, com precipitação média anual de $1.442 \mathrm{~mm}$. $\mathrm{O}$ clima da região é subtropical úmido, sem estação seca. A temperatura média em janeiro é de $22^{\circ} \mathrm{C}$ e a de julho fica em torno de $10^{\circ} \mathrm{C}$, com mais de cinco geadas por ano. Numa pequena extensão, a vegetação primitiva é constituída de floresta pluvial subtropical, e em sua grande maioria de floresta de pinhais (formação de araucária). Atualmente, a área plantada é distribuída de forma que a floresta natural possui 1.659 ha, predominando Arancaria angustifolia (pinheiro-do-Paraná) e Ocotea porosa (imbuia). Quanto a área plantada constituída de 1.273 ha, existem 776 ha de Pinus elliotii, 603 ha de A. angustifolia, pequenas ilhas de Eucaliptus sp., e o restante de outras espécies diversas (IBAMA 1993).

\section{Metodologia}

A metodologia das capturas foi adaptada de GREENHALL \& PARADISO (1968) e de REIS \& PERACCHI (1987). As coletas foram realizadas durante as três primeiras horas da noite e excepcionalmente nos lugares de repouso dos morcegos. Foram usadas redes de espera para as coletas, armadas em clareiras, transversalmente em estradas e trilhas dentro da mata e sobre pequenos riachos. Como o interesse é comparar as coletas do Alto, Médio e Baixo Tibagi foi utilizado o mesmo esforço de captura em cada área de estudo, ou seja, $174 \mathrm{~m}^{2}$ de rede durante o período de 1982 (outono, inverno, primavera, verão), 1993 (inverno e primavera) e 1994 (outono e inverno), totalizando $1.392 \mathrm{~m}^{2}$ de rede em 24 horas de coleta em cada local. Foram acrescentadas paralelamente às coletas do Baixo Tibagi, trabalhos de levantamento realizados de 1982 a 1991 para um maior detalhamento deste trabalho.

Somente dois exemplares de cada espécie foram sacrificados e fixados em formol a $10 \%$ e conservados em álcool $70 \%$, seguindo os critérios de VIZOTO \& TADDEI (1973). Após a obtenção de dados pertinentes, outros foram coletados, tais como: peso, sexo, estágio reprodutivo, e coleta de fezes, os demais exemplares capturados eram novamente soltos no seu local de origem.

Para analisar a diversidade da vegetação dos três locais de estudo, SOARES (1993) utilizou o índice de diversidade de Shannon-Wiener (H') (KreBs 1989).

\section{RESULTADOS E DISCUSSÃO}

Do número total de espécies existentes em uma comunidade, uma porcentagem é geralmente dominante (espécies comuns) e uma grande porcentagem é representada por um pequeno número (espécies raras) (ODUM 1985). Excluindo os molossídeos que normalmente não são capturados em redes pelo seu tipo de comportamento, observa-se que Artibeus lituratus (35,8\%), Carollia perspicillata $(18,2 \%)$, Sturnira lilium (11,5\%), Myotis nigricans (11,5\%) e Desmodus rotundus $(4,8 \%)$ representam $81,8 \%$ de todos os indivíduos coletados (Fig. 2). As outras 16 espécies apenas $18,2 \%$.

A ocorrência disto se dá pelo ajuste entre os organismos e seu ambiente, definido pelo grau de adaptação de cada espécie (PIANKA 1982). A adaptação ocorre em várias dimensões, onde os organismos devem se encaixar simultaneamente aos 


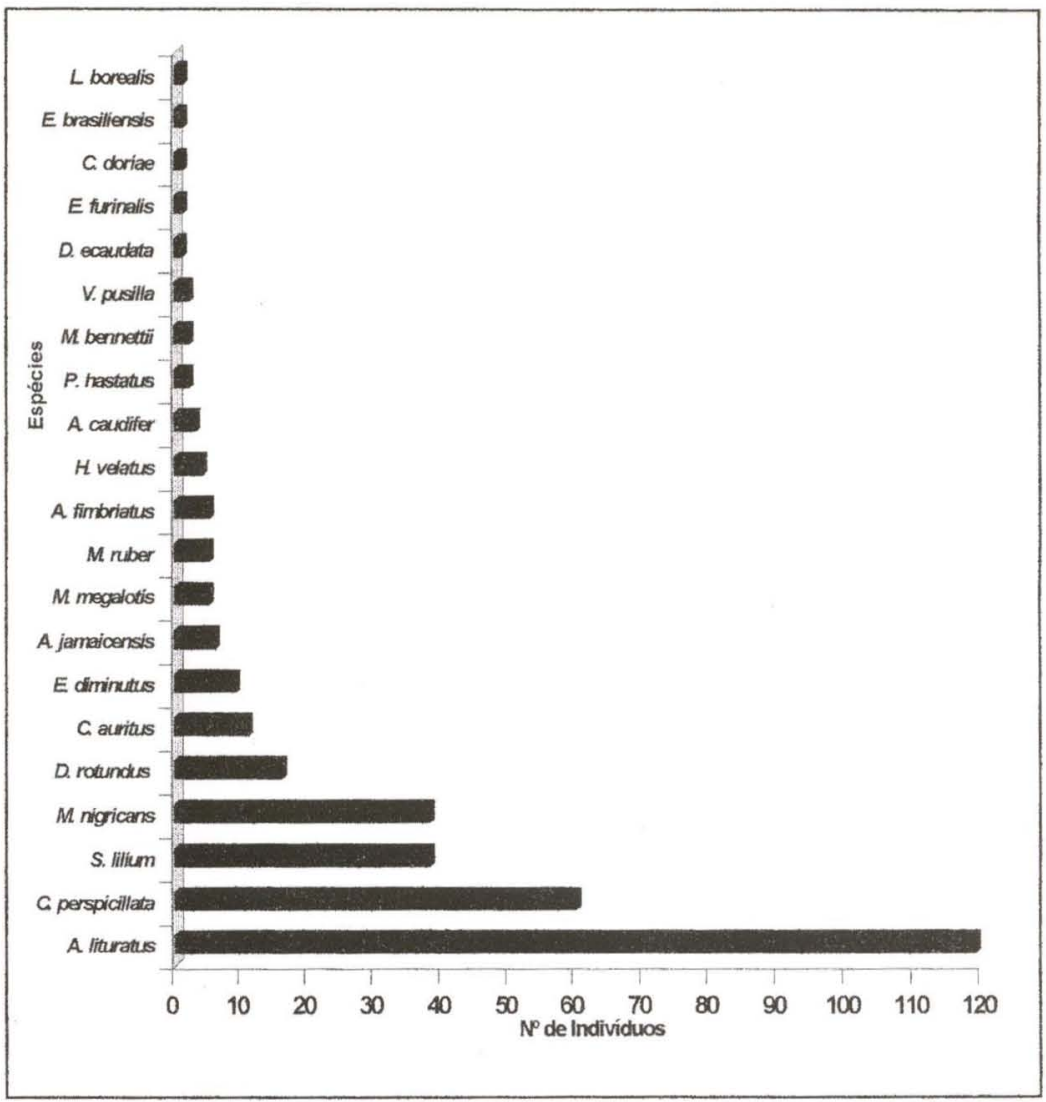

Fig. 2. Número total de espécies e individuos coletados nos três locais de estudo de 1992 a 1994.

padrões de temperatura, umidade, competidores, predadores e encontrar uma situação média, respeitando os seus limites. Assim sendo, as 16 espécies pouco freqüentes nas capturas podem se acomodar nos ambientes estáveis e sem mudanças bruscas. As alterações rápidas, na maioria das vezes, provocadas pelo homem, podem inclusive ameaçá-las, como está acontecendo com M. ruber, C. doriae, (ameaçadas) P. bilabiatum, M. levis e R. tumida (presumivelmente ameaçadas) na Bacia do Rio Tibagi (Tab. I). Ainda que FISHER (1930) tenha dito que nenhum organismo está perfeitamente adaptado, vimos que as cinco espécies abundantes estão melhor adaptadas nos seus ambientes em um maior número de dimensões, toleram grandes alterações ao contrário das outras mais especializadas com capacidades homeostáticas limitadas, mas também importantes para o equilíbrio do ecossistema onde vivem. O risco é que espécie com alto potencial adaptativo, como Artibeus lituratus, invade o habitat às custas de espécies endêmicas sensíveis. Com 
isso, a riqueza total de espécies diminui e a biota fica homogeneizada, uma vez que, espécies comuns ocupam os espaços. Fato constatado pela equipe após 24 anos de coletas em regiões tropicais.

Sabe-se que fatores intensos de natureza física ou biológica predispõem a diminuição da diversidade (REIS \& MULLER 1995). Como exemplo tem-se a Floresta Nacional do Irati, com 3.571 ha com uma área reflorestada de 1.273ha de Pinus elliotii e P. talda, Araucaria angustifolia e Eucaliptus sp. (IBAMA 1993) onde ocorreram as coletas, de apenas nove espécies, sendo cinco consideradas aqui como raras, ou seja, com pequeno potencial adaptativo, muito sensíveis e pouco representativas nas coletas, mesmo usando diversos métodos de captura, durante longo tempo. No Parque Estadual Mata dos Godoy, com uma área de apenas 680 ha, mas constituído de uma floresta tropical intocada, observa-se no mesmo período de coletas, 15 espécies, sendo 10 delas tidas como raras. É claro que uma floresta tropical com numerosos nichos, pela diversidade elevada de flora favorecem oportunidades para um maior número de espécies, como sugere (ODUM 1985) e conseqüentemente podem apresentar uma diversidade elevada (TADDEI \& PEDRO 1998).

Comparando-se a riqueza de espécies vegetais de áreas remanescentes ao longo da bacia do Rio Tibagi, através do índice de Shannon-Wiener (H'), observouse que a diversidade florística aumenta do Alto para o Baixo Tibagi (SOARES 1993). Na remanescente da Fazenda Doralice, localizada no mesmo paralelo do Parque Estadual Mata dos Godoy (Baixo Tibagi), o índice de diversidade foi de $\mathrm{H}^{\prime}=1.66$, enquanto outra próxima à Floresta Nacional de Irati (Alto Tibagi) foi de $\mathrm{H}^{\prime}=0.89$; e no médio, a Reserva Biológica da Klabin tem-se $H^{\prime}=1.58$, confirmando com os dados da Ordem Chiroptera nesses locais.

Os resultados mostram que fatores limitantes não estão restritos aos físicos. As interações das relações biológicas dos morcegos como a diversidade vegetal, tamanho e qualidade da área são tão importantes no controle da distribuição e abundância dos organismos como outras. Quando o homem intervém com ações intensas em uma comunidade natural, as conseqüências são rápidas (WILSON 1997).

Sendo este trabalho realizado em "Unidades de Conservação" era de se esperar que reservas maiores, apresentassem maior riqueza, com áreas para preservar populações viáveis de espécies nativas de morcegos, sem o risco de desaparecimento. Em geral acredita-se que quanto maior uma área, maior o número de espécies, ou seja, como sugere DiAMOND (1980) quando se aumenta em 100\% o tamanho da área, o número de espécies, em média aumenta $25 \%$. Mas, segundo LYNCH \& WHIGHAM (1984), a melhor estratégia de preservação das espécies, com certeza é não alterar a qualidade total do habitat de procriação (sendo isso mais importante do que enfatizar a extensão de cada reserva). Acredita-se como ZIMMERMAN \& BIERREGAARD (1986) que uma unidade de conservação grande sem bons criadouros, abrigaria um número menor de espécies, do que uma pequena, com fontes de água e outros recursos acolhedores e de subsistência. Contudo, uma reserva estreita em forma de faixa poderia até não abrigar espécies raras, pois o vento, a chuva, a vegetação invasora e o barulho - os chamados efeitos de borda (LEWIN 1984) estariam fazendo com que as espécies mais sensíveis parassem de se reproduzir ou mesmo desaparecessem. 
Tabela I. Morcegos encontrados nos diferentes fragmentos florestais coletados (unidades de conservação).

\begin{tabular}{|c|c|c|c|c|c|}
\hline Familia/Subfamilia & Espécies & $\begin{array}{l}\text { Status de } \\
\text { conservação }\end{array}$ & $\begin{array}{l}\text { Baixo Tibagi } \\
\text { Godoy }\end{array}$ & $\begin{array}{c}\text { Médio } \\
\text { Tibagi Klabin }\end{array}$ & $\begin{array}{l}\text { Alto Tibagi } \\
\text { Irati }\end{array}$ \\
\hline \multicolumn{6}{|l|}{ Noctilionidae } \\
\hline & Noctilio albiventris Desmarest, 1818 & & * & & \\
\hline \multicolumn{6}{|l|}{ Phyllostomidae } \\
\hline & Chrotopterus auritus (Peters, 1856) & & 5 & 4 & 2 \\
\hline \multirow[t]{3}{*}{ Phyllostominae } & Micronycteris megalotis (Gray, 1842) & & 3 & 2 & \\
\hline & Mimon bennettii (Gray, 1838) & & * & 1 & 1 \\
\hline & Phyllostomus hastatus (Pallas, 1767) & & * & & \\
\hline \multirow[t]{2}{*}{ Glossophaginae } & Anoura caudifer (E. Geoffroy, 1818) & & * & 3 & \\
\hline & Glossophaga soricina (Pallas, 1766) & & * & & \\
\hline Carollinae & Carollia perspicillata (Linnaeus, 1758) & & $* 50$ & 4 & 6 \\
\hline \multirow[t]{8}{*}{ Stenodermatinae } & Artibeus lituratus (Olfers, 1818) & & $* \quad 45$ & 43 & 30 \\
\hline & Artibeus fimbriatus Gray, 1838 & & 4 & & 1 \\
\hline & Artibeus jamaicensis Leach, 1821 & & 5 & & 1 \\
\hline & Chiroderma doriae Thomas, 1891 & Ameaçada & 1 & & \\
\hline & Platyrihinus lineatus (E. Geoffroy, 1810) & & 2 & & \\
\hline & Pygoderma bilabiatum (Wagner, 1843) & $\begin{array}{l}\text { Presumivelmente } \\
\text { ameaçada }\end{array}$ & * & & \\
\hline & Sturnira lilium (E. Geoffroy, 1810) & & 19 & 9 & 10 \\
\hline & Vampyressa pusilla (Wagner, 1843) & & 2 & & \\
\hline \multirow[t]{2}{*}{ Desmodontinae } & Desmodus rotundus ( $E$. Geoffroy, 1810) & & * 13 & 3 & \\
\hline & Diphylla ecaudata (Spix, 1823) & & 1 & & \\
\hline \multicolumn{6}{|l|}{ Vespertilionidae } \\
\hline & Eptesicus brasiliensis (Desmarest, 1819) & & * & 1 & \\
\hline & Eptesicus diminutus Osgood, 1915 & & * & 5 & 4 \\
\hline & Eptesicus furinalis (d'Orbigny, 1847) & & 1 & & \\
\hline & Histiotus velatus (I. Geoffroy, 1824) & & * & 4 & \\
\hline & Lasiurus borealis (Muller, 1776) & & 1 & & \\
\hline & Myotis levis (I. Geoffroy, 1824) & $\begin{array}{l}\text { Presumivelmente } \\
\text { ameaçada }\end{array}$ & * & & \\
\hline & Myotis nigricans (Schinz, 1821) & & 12 & 16 & 10 \\
\hline & Myotis ruber (E. Geoffroy, 1806) & Ameaçada & * & 5 & \\
\hline & Rogheessa tumida H. Allen, 1866 & $\begin{array}{l}\text { Presumivelmente } \\
\text { ameaçada }\end{array}$ & * & & \\
\hline \multicolumn{6}{|l|}{ Molossidae } \\
\hline & Molossops abrasus (Temminck, 1827) & & * & & \\
\hline & Molossus ater E. Geoffroy, 1905 & & * & & \\
\hline & Molossus molossus (Pallas, 1766) & & * & & \\
\hline & Nyctinomops laticaudatus (E. Geoffroy, 1805) & & * & & \\
\hline & Tadarida brasiliensis (I. Geoffroy, 1824) & & * & & \\
\hline & $\mathrm{N}^{0}$ individuos & & 164 & 100 & 65 \\
\hline & $N^{\circ}$ espécies & & $32 \quad 15$ & 13 & 9 \\
\hline
\end{tabular}

Observação: esforço de captura: $174 \mathrm{~m}^{2} / 24$ horas de coleta $\left(264 \mathrm{~m}^{2}\right)$.

*) Espécies coletadas fora deste esforço de captura de 1982 a 1991 - Baixo Tibagi (P.E. Mata dos Godoy).

A fragmentação dos habitats vem continuamente ocorrendo em decorrência do crescimento econômico e, conseqüentemente, as espécies cujas necessidades são maiores (espécies raras), fatalmente desaparecem, pois nem todos os morcegos podem ser tratados da mesma maneira, tendo em vista que se trata de uma ordem com 147 espécies no Brasil, com comportamentos e necessidades diferentes. MYERS (1979 apud LUGO 1997) afirma que o mundo estava perdendo uma espécie por dia nos anos 70. Nos meados dos anos 80, aumentou para uma espécie por hora e no final do século nosso planeta perderá entre 20 a 50\% das espécies. O que já não perdeu-se com a destruição de $95 \%$ das florestas do Paraná? 


\section{REFERÊNCIAS BIBLIOGRÁFICAS}

Di^mond, J.M. 1980. Subdivision of nature reserves and the maintenance of species diversity. Nature 285: $567-658$.

FISHER, R.A. 1930. The genetical theory of natural selection. Oxford, Clarendon, 272p.

Greenhall, A.M. \& Paradiso. 1968. Bats and bat banding. Washington, D.C., Bureau of Sport Fisheries and Wildlife Resource Publication 72, 47p.

HANDley JR., C.O. 1966. Check list of the mammals of Panama, p. 753-795. In: R.L. WEnzEL \& V.J.

TIPTON (Eds). Ectoparasites of Panama. Chicago, Feild Mus. Nat. Hist., 861p.

Huber, J. 1910. Matas e madeiras amazônicas. Bol. Museu Paraense Emilio Goeldi 6: 91-225.

HuMPhREy,S.R. \& F.J. BonacCorso. 1979. Population and community ecology, p. 409-441. In: R.J.

Baker, R.J. J.K. Jones JR. \& D.C. CARTER (Eds). Biology of bats of the New World family

Phyllostomidae. 3. Spec. Publ. Mus. Texas Tech. Univ., Lubbock, 16, $441 \mathrm{p}$.

IвAMA. 1993. Floresta Nacional de Irati. Curitiba, Ibama, 3p.

KREBS, C.J. 1989. Ecological methodology. New York, Harper \& Row publishers, 654p.

LEwIN, R. 1984. Parks: How Big is enough? Science 225: 611-612.

LYNCH, J.F. \& D.F. WHIGHAM. 1984. Effects of forest fragmentation on breeding bird communities in Maryland, U.S.A. Biol. Conserv. 28: 287-324.

LuGo, A. E. 1997. Estimativas de reduções na diversidade de espécies da floresta tropical, p. 72-87. In:

E.O. Wilson (Ed.). Biodiversidade. Rio de Janeiro, Nova Fronteira, 657p.

NowaK, R.M. 1991. Walker's Mammals of the World. London, The Johns Hopkins University Press, Vol. $1,5^{\text {th }}$ ed., 642 p.

ODUM, E.P. 1985. Ecologia. Rio de Janeiro, Interamericana, 434p.

PIANKA, E.R. 1982. Ecologia Evolutiva. Barcelona, Omega, 365p.

Reis, N.R. \& A.L. Pfracchi. 1987. Quirópteros da região de Manaus, Amazonas, Brasil (Mammalia, Chiroptera). Bol. Mus. Paraense Emílio Goeldi, série Zool., 3 (2): 161-182.

REIS, N.R.\& M.F. MULLER. 1995. Bat diversity of forest and open areas in a subtropical region of South

Brazil. Ecologia Austral 5: 31-36

SOARES, F.S. 1993. Aspectos da fauna e da flora da bacia do Rio Tibagi. Londrina, UEL/COPATI/KLABIN, Vol. 2, $3^{\circ}$ Relatório Técnico Científico, 76p.

Taddei, V.A. \& W.A. Pedro. 1998. Morcegos (Chiroptera, Mammalia) do Vale do Ribeira, Estado de

São Paulo: Diversidade de espécies. Anais VIII Seminário Regional de Ecologia 8: 835-836

Trewartha, G.T. \& L.H. Horn. 1990. An introduction to climate. New York, McGraw-Hill, 416p.

VAN DFR PIJ., L. 1957. The dispersal of plants by bats (Chiropterocory). Acta Botanica Neerlandica 6: 291-315.

Vizoto, L.D. \& V.A. TADDEI. 1973. Chave para determinação de quirópteros brasileiros. Bol. Ciências 1: $1-72$.

Zimmerman, B.A. \& R.O. BilrregaArd. 1986. Relevance of the equilibrium theory of island biogeography and species-area relations to conservation with a case from Amazonia. Jour.

Biogeogr. 13: 133-143.

Wilson, E.O. 1997. Biodiversidade. Rio de Janeiro, Nova Fronteira, 657p.

Recebido em 29.X.1998; aceito em 23.VIII.2000. 